\title{
Correction to: Biomechanics of Stabilizing the Keratoconic Cornea
}

\author{
Cynthia J. Roberts
}

\section{Correction to:}

Chapter 9 in: A. Barbara (ed.), Controversies in the Management of Keratoconus, https://doi.org/10.1007/978-3-319-98032-4

This chapter was inadvertently published with incorrect author group. The correct author group and the affiliation is as follows:

Cynthia J. Roberts

Martha G. and Milton Staub Chair for Research in Ophthalmology

Professor of Ophthalmology and Vision Science

Professor of Biomedical Engineering

The Ohio State University

Columbus, Ohio

USA 